\section{CASTING CELLS}

Stephen W. Carmichael, ${ }^{1}$ Mayo Clinic carmichael.stephen@mayo.edu

It's always refreshing to read about two old techniques being combined in a novel way to yield new information. Osamu Ohtani and Yuko Ohtani recently accomplished this. ${ }^{2}$ Louis Ranvier probably introduced the use of silver nitrate as a histology stain over a century ago. Whereas Ranvier is best known for describing nodes along the nerve fiber, he also demonstrated that silver nitrate stained endothelial cells, allowing their borders to be clearly visualized. In the 1970's, Takuro Murakami first used the scanning electron microscope (SEM) to examine corrosion casts of the vasculature. Much of what we currently know about the microarchitecture of blood vessels has been discovered using this technique. Ohtani and Ohtani have combined these two techniques to visualize the outline of endothelial cells on vascular casts of arteries, veins, and capillaries.

They began by perfusion-fixing rats through the thoracic aorta. This was followed by an aqueous solution of silver nitrate, followed by glucose for osmotic stability. Some rats were then perfused with a photographic developer to deposit silver halide. This worked too well, because the silver halide was apparently so abundant that subsequent perfusion was not possible. What worked better was to follow the silver nitrate and glucose perfusions with a casting medium (Mercox). The tissues selected for study were excised and either exposed to a strong light, or immersed in the photographic developer. After the Mercox had completely polymerized, the tissue was corroded away with sodium hydroxide and then rinsed. Wet casts could simply be transferred to a low-vacuum SEM with a cooling stage. The images of the wet casts were al'most identical in quality to backscattered micrographs of dried casts without any coating. But the best images appeared to be of dried casts that were coated with metal and imaged by SEM in the secondary electron mode.
The specimens that were exposed to strong light produced good images near the lighted surface, but immersion in developer worked best.

Ohtani and Ohtani published elegant micrographs of casts of different sized vessels. And on these casts that demonstrated the three dimensional arrangement of the vessels, one could clearly see the location of the outlines of the endothelial cells. Examination at higher magnification showed that silver grains were deposited in the grooves along the borders between cells. Also, at sufficiently high magnification, impressions of the nuclei could be discerned. Occasionally, for no apparent reason, the boundaries were not marked, but most of the time the method worked very well.

The SEM images were consistent with what is known from studies with the light microscope. Endothelial cells lining arteries and arterioles had an elongated spindle shape with undulating margins. Capillary endothelial cells were also fusiform, but had a more irregular shape. Venules and veins had lining cells that were about as long as cells on the arterial side, but were distinctly wider and less undulated. It was clearly demonstrated that variations in endothelial cell sizes and shapes could be seen with this method.

This elegant combination of two established techniques allows for simultaneous demonstration of the three dimensional microarchitecture of blood vessels and the size and shape of the cells lining the vessels. This method will be a powerful tool for examining endothelial cell morphology and microvascular organization in virtually any organ, under both normal and pathologic conditions.

1. The author gratefully acknowledges Professor Osamu Ohtani for reviewing this article.

2. Ohtani, $\mathrm{O}$. and $\mathrm{Y}$, Ohtani, $\mathrm{A}$ corrosion casting/scanning electron microscope method that simultaneously demonstrates clear outlines of endothelial cells and three-dimensional vascular organization, Arch. Histol. Cytol. $63: 425-429,2000$.

\title{
INDEX OF ARTICLES
}

Casting Cells Stephen W. Carmichael, Mayo Clinic

Microscopy For Children: A Bibliography Update, April 2001 ...6 Caroline Schooley, MSA Project MICRO Coordinator

The Development of an X-ray Spectral Database

For-Forensic Science

Dennis Ward, Federal Bureau of Investigation

A Simple Technique For The Removal Of Plant Cell.............. 14

Protoplasm To Facilitate Scanning Electron Microscopy

Of Fungal Haustoria And Plant Cell Wall Features

B.A. Richardson \& C.W. Mims, University of Georgia

Notes On Vacuum Techniques For Microscopists.

Scott D. Walck, PPG Industries

New And/Or Interesting in Microscopy at PITTCON 2001 .....20

Tips On Focusing Transmission Electron Microscopes .........22 Steve Chapman, Protrain

Copper Contamination of Gunshot Residue and Other .........24

Particle Spectra

Bryan R. Burnett, Meixa Tech

A Method for Decapsulting Integrated Circuit Chips

Becky Holdford, Texas Instruments, Inc.
Diffusion Pumps and Water Chillers F.C. Thomas, Geological Survey of Canada (Atlantic)

How Do I Visualize A Thin Gold Film On A Smooth Surface . 30 Mary Mager, University of British Columbia

Precision Attachment of (Silica) Spheres to AFM 32

Cantilever Tips

Shane Huntington \& Ms. Sarah Nespolo

University of Melbourne

Letters To The Editor: Resolution And Resolving Power ....... 34

Ceramic Powder Preparation for EM . Charles Garber, SPI Supplies

Cleaning a Calibration Standard Gold........

Rick Workman, University of Arizona

Serial Sections for TEM Without Using Glue....................... 36 Timothy G. Schneider, Thomas Jefferson University

Embedding Vibratome ${ }^{T M}$ Sections for TEM ......................... 36 John Chandler, Colorado State University

A Brief Tip On Decolorizing DAB Staining for 37 Immunohistochemistry Mike King, University of Florida College of Medicine 


\section{The Biolmaging Leader}

MetaMorph

Software and Systems for:

6-D Imaging

Brightness Measurements

- Colocalization

- Fluorescence Recovery After Photobleaching (FRAP)

- Fluorescence Resonance Energy Transfer (FRET)

- Gene Chips \& Microarrays

Immunofluorescence

Live/Dead Assays

- Green Fluorescent Protein

- Intracellular Calcium, pH \& Magnesium

Motion Analysis

Morphometry

Ratio Imaging

- Time Lapse

... and many more applications!

Join Universal Imaging's Education Team at the 2001 Analytical and Quantitative Light Microscopy Course.

Place: Marin Biological Laboratory, Woods Hole, MA

Dates: May 10 - May 18

Directors: Greenfield Sluder and David Wolf, University of Massachusetts Medical Center, Worcester.

Working to Improve Your Image

COMMERCIAL

BEST SOFTWARE by Cell Biologists*

"MME survey of attendees at the American Society for Cell Biology Annual meeting December 2000 
- Housing at M\&M 2001:

To make reservations for Microscopy \& Microanalysis 2001 on line, visit:

www.pkghirss.com/events $/ \mathrm{mm} 2001 / \mathrm{mm} 2001$.html

To fax your reservations, simply download the housing form from the above site and fax it to (562)590-9366. Phone number for questions (they will not take reservations over the phone) is (562)436-3645, ext. 111.

- Microscopy \& Imaging Resources on the WWW, a revised and updated version, is now available at a new URL.

The goal of this site has always been to provide resources for University students, staff and faculty who want to learn more about microscopy and imaging. The site includes K-12 educational links, information on light microscopy, histology, confocal microscopy, fluorescence techniques, electron microscopy, and digital imaging.

URL: http://swehsc.pharmacy.arizona.edu/exppath/

- International 11-Day Short Course on 3D Microscopy of Living Cells will be held on 18/28 June 2001 at the University of British Columbia, Vancouver, BC., Canda.

Modern methods of 3D light microscopy promise a revolutionaly improvement in our ability to view living cells. To help convert this promise to reality for a wider selection of biological scientists, an intensive eleven-day residential course concentrating on all aspects of 3D Microscopy of Living Cells will be held. The course includes 4 days on $2 D$ techniques, 6 days of $3 D$ techniques and a summary presentation. It covers basic microscopy to the highest level of confocal microscopy. A half-day pre-course is offered for any who may need to bursh up on basic optics.

For more information:

http://www.cs.ubc.ca/spider/ladic/course/bulletin.html

\section{- Yale School of Medicine Features Exhibit on Microscopy.}

As part of the tercentennial of Yale University, the Yale School of Medicine will feature an exhibit on "Microscopy: Tools Of The Biomedical Sciences" from 26 April to 4 June, 2001. For more information, contact Carl Zeiss, Inc. at: www.zeiss.com/micro

28th Annual Meeting of the Microscopical Society of Canada will be held on 6/8 June 2001 at the University of New Brunswick, Fredericton, New Brunswick, Canada.

The scientific program features invited speakers from the environmental sciences, plant sciences, material sciences, EELs applications, confocal microscopy and magnetic resonance imaging (MRI). Workshops will focus on specimen preparation techniques for both the biological and material sciences, including ultramicrotomy, polishing methods, high-pressure freezing techniques and a workshop on preparation of digital images for publication. Vendors and manufacturers will be demonstrating the latest developments in the field of microscopy.

All information and required forms for registration are available at the conference website: http://unb.ca/msc2001
In the upcoming Microscopy Society of America's M\&M Conference in Long Beach, CA (5/9 August 2001), we will again hold a "Just For Fun Micrograph Contest".

The concept of the contest is based upon composite images, each made up of two or more other images, at least one of which must be microscopical in nature. Contestants may enter up to two images each and do not have to be present at the MSA Conference to win.

Entrees will be displayed in our booth and conference attendees will be invited to vote on which they consider to be most "creative and interesting". First prize will be $\$ 300.00$ and the second and third prizes, respectively, will be $\$ 200.00$ and $\$ 100.00$. All contestants may received two microscopy prints by David Scharf. And winning entries may be featured as covers on Microscopy Today.

Entries must, of course, be on hard copy. While any reasonable size is acceptable, a size of around $8^{n} \times 11^{\prime \prime}$ is recommended. Entries should be on rather stiff background suitable for posting. A $3^{\prime \prime} \times 5^{n}$ card should accompany each entry - with an image description and contributors name and affiliation.

Entries are welcome from all interested in microscopy: "users" as well as manufacturers/suppliers. And, of course, those overseas as well as those "local".

And, should you decide to enter our contest, it would be helpful if you so advise (with the number of your entries) - just to be sure that we have proper display space for all entries. You might advise by ernail (microtoday@mindspring.com) or by fax (608-836-1969).

I hope that you will decide to enter our contest! . Don Grimes, Microscopy Today

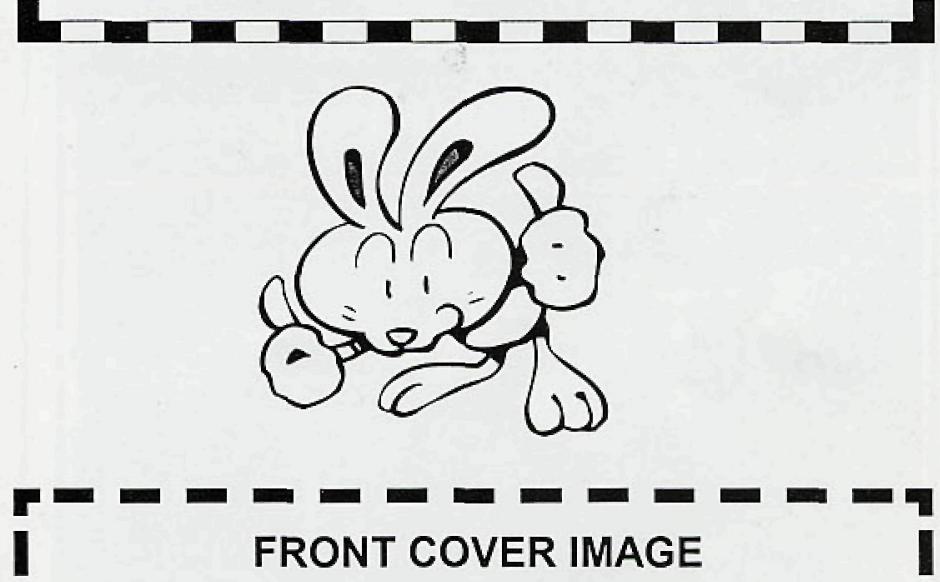

This is a scanning electron micrograph of a sporangium of the plasmodial slime mold (or Myxomycete) Physarum polycephalum. The actual size is about $3 \mathrm{~mm}$ tall. The yellow spore head contains $10 \mu \mathrm{m}$ diameter spores, which are airdispersed. Some of these spores can be seen at the edges of the sporangium. When they fall on a moist substratum, they germinate to release motile myxamoebae. These later fuse to produce the macroscopic yellow plasmodium. When exposed to light and drying, the plasmodium forms multiple sporangia like the one shown. A single sporangium was attached to a standard SEM stub, gold coated and photographed with a Hitachi S-450 SEM. The negative was scanned with an Epson 1600 scanner and then colorized using Adobe PhotoDeluxe.

Image courtesy of Henry Aldrich, University of Florida 